\title{
Philosophiques
}

\section{Les travaux de jeunesse de Hegel et l'interprétation de sa philosophie de la religion}

\section{Benoît Garceau}

Volume 1, numéro 1, avril 1974

URI : https://id.erudit.org/iderudit/203002ar

DOI : https://doi.org/10.7202/203002ar

Aller au sommaire du numéro

Éditeur(s)

Société de philosophie du Québec

ISSN

0316-2923 (imprimé)

1492-1391 (numérique)

Découvrir la revue

Citer cet article

Garceau, B. (1974). Les travaux de jeunesse de Hegel et l'interprétation de sa philosophie de la religion. Philosophiques, 1(1), 21-49.

https://doi.org/10.7202/203002ar
Résumé de l'article

Cet article vise à montrer que, malgré le peu d'importance que les études récentes de la philosophie hégélienne de la religion accordent aux écrits antérieurs à la Phénoménologie, l'analyse des Jugensdschriften reste indispensable : a) pour comprendre la genèse de la thèse, centrale dans la Religionsphilosophie, sur la différence entre la raison et l'entendement, b) pour reconnaître que la logique de l'absolu employée par la théologie hégélienne fut davantage le résultat de l'attachement de Hegel à son idéal de raison et de sa critique du Kantisme, que le fruit de sa méditation du christianisme ; c) pour découvrir l'unité du développement de la pensée hégélienne. 


\title{
LES TRAVAUX DE JEUNESSE DE HEGEL
}

\section{ET \\ L'INTERPRÉTATION DE SA PHILOSOPHIE DE LA RELIGION}

\author{
par Benoît Garceau
}

Malgré la prolifération des travaux sur les écrits de jeunesse de Hegel, l'accord n'est pas encore fait sur la place à donner à ces écrits dans la lecture de l'ensemble de son oeuvre. S'il est vrai que la découverte par Dilthey et l'édition par Nohl des fragments de Tubingue, Berne et Francfort, ont levé le voile sur ses premières préoccupations, presqu'exclusivement religieuses, et ont ouvert la porte à des études toujours mieux documentées de la genèse de sa pensée, il reste que la tendance des travaux sur Hegel a été de mettre en doute l'importance de la lecture de ces textes «théologiques » pour l'intelligence de l'ensemble du système hégélien.

C'est surtout dans l'étude de la philosophie hégélienne de la religion, dont l'essor a été remarquable au cours des dernières années, que l'on a pris la décision d'attendre peu de secours des Jugendschriften. Les travaux de Bruaire ${ }^{1}$, Fackenheim ${ }^{2}$, Cha-

1. «Pour cette compréhension requise (de la philosophie hégélienne), suivre la genèse de la pensée, en retracer l'histoire, répérer les préalables du système organisé n'offre guère de secours. C'est en essayant de saisir l'oeuvre dans son unité achevée et terminale que nous pouvons espérer trouver une réponse décisive ». C. BRUAIRE, Logique et religion cbrétienne dans la philosopbie de Hegel, Paris, Seuil, 1964, p. 9.

2. «Three conclusions follow from these considerations. First, the Early theological Writings are important for Hegel's biography; they add little to the understanding of his mature thought, beyond confirming that, if such thinkers as Marx and Kierkegaard, attack Hegel, it is not because he is irrelevant or absurd, but rather because he is too close for comfort. Next, the 
pelle $^{3}$, Theunissen ${ }^{4}$, Léonard ${ }^{5}$, s'inspirent tous d'une même stratégie: chercher à comprendre Hegel à la lumière de son système, plutôt qu'en reconstruisant la genèse de sa pensée; renoncer à attendre de l'exploration des écrits de jeunesse une lumière que les oeuvres de la maturité ne révéleraient pas. Sans nier leur valeur pour la biographie intellectuelle du philosophe, on n'estime pas la lecture des Jugendschriften indispensable à la compréhension de sa philosophie définitive de la religion ${ }^{6}$,

hegelian philo-ophy, more than any other, requires systematic rather than genetic exposition. Finally, there is no legitimate way of circumventing the fundamental mystery of the mature system ». E.L. FACKENHEIM, The Religious Dimension in Hegel's Thought, Bloomington/London, 1967, p. 7.

3. «Par souci de fidélité à la pensée hégélienne, qui s'est voulue et comprise comme système, nous renonçons donc à toute méthode génétique d'interprétation. Nous refuserons de citer, sinon à titre exceptionnel et doxographique, les oeuvres antérieures à la Phénoménologie. Les Jugtndschriften sont d'ailleurs, on le sait, l'oeuvre d'une époque où l'Aufbebung hégélienne de la religion se cherche encore sous les traits d'une critique politique et moralisante que le système ultérieur dépassera en les englobant ». A. CHAPELLE, Hegel et la religion, Paris, Editions universitaires, 1964, vol. I, p. 12.

4. M. THEUNISSEN, Hegels Lebre vom absoluten Geist als theologischpolitiscber Traktat, Berlin, Walter de Gryter, 1970, 459 p.

5. A. LEONARD, La foi chez Hegel, Paris, Desclée, 1970, 426 p. C'est par les oeuvres de jeunesse de Hegel que Léonard introduit la problématique hégélienne de la foi et c'est par elles qu'il cherche à obtenir la pré-compréhension de l'objet de son enquête, mais il renonce à étudier ces oeuvres pour elles-mêmes et ne commente que les textes qui prépatent à comprendre les écrits systćmatiques de la maturité. Dans le programme de lecture qu'il suggère aux jeunes philosophes, Comment lire Hegel. Considérations spéculatives et pratiques, in Revue pbilosopbique de Louvain, 70(1972), 573586, la lecture des Jugendschriften n'est rccommandée qu'à titre de complément à l'initiation à Hegel, après que le néophyte hégélien aura parcouru les oeuvres systématiques (voir p. 585).

6. Les deux auteurs qui ont le plus insisté sur l'importance des Jugendschriften pour la comprćhension du discours hégélien sur la religion sont: P. ASVELD, La pensée religieuse du jeune Hegel. Liberté et aliénation, Louvain, 1953, p. 182 : «il est fort important, lorsqu'on s'occupe de la philosophie religieuse de la maturité, de ne pas perdre de vue les écrits de jeunesse, faute de quoi on risque de se laisser prendre au mirage des mots, de prendre à la lettre l'exposé de la religion absolue et d'y voir autre chose que l'expression symbolique de vérités philosophiques », et G. ROHRMOSER, Théologie et aliénation dans la pensée du jeune Hegel, Paris, Beauchesne, 1970, p. 20 : «S'il est légitime d'expliquer Hegel par lui-même, s'il est vrai que, en se systématisant, l'idéal des années de jeunesse non seulement devient systèrne de la réflexion mais aussi y a trouvé son achèvement. la compréhension du point de départ, développé dans les écrits de jeunesse, est décisive pour l'interprétation de la philosophie hégélienne dans son ensemble $\gg$. 
et on refuse surtout d'y voir le meilleur de Hegel que le système aurait ensuite stérilisé ${ }^{7}$.

Cette décision de soumettre Hegel à une lecture exclusivement systématique, à partir des oeuvres où sa pensée religieuse s'exprime de façon achevée, semble, à première vue, s'appuyer sur un choix légitime: celui de chercher à comprendre Hegel bégéliennement, comme il s'est compris lui-même, à la lumière de son propre système, plutôt que vouloir trouver son secret dans les données lacunaires des fragments de jeunesse, dont la réorganisation a trop souvent été liée aux hypothèses et aux présupposés des interprètes. A la lecture génétique, souvent reprise depuis Dilthey, et qui a abouti à des portraits divergents du jeune Hegel et de son attitude à l'égard de la religion selon les grilles de lecture utilisées par les chercheurs ${ }^{8}$, il était sans doute recommandable de substituer une lecture systématique, dont le seul a priori serait la structure du système hégélien.

Une raison plus profonde cependant paraît justifier cette stratégie. La théologie spéculative développée dans la Pbénoménologie, l'Encyclopédie et les Cours de Berlin sur la religion, s'appuie sur un présupposé fondamental, dont on ne trouve aucune trace dans la critique de la religion exposée dans les Jugendscbriften. C'est un fait, confirmé par l'étude récente de

7. Voir A. LEONARD Hegel et la foi, p. 17, note 2. - On a un bon exemple de l'attrait produit chez certains interprètes par les travaux de jeunesse de Hegel dans l'ouvrage de W. KAUFMANN, Hegel. A Reinterpretation, New York, Doubleday, 1965, 415 p. Voir en particulier p. 291 : «But it is a fact that Hegel did his most original work before he went to Berlin and became a famous professor, that his inspiration gradually dried up, and that his growing conservatism, went together with a lack of new ideas 》.

8. Le jeune Hegel de W. KAUFMANN, op. cit., est irreligieux dès les années de Tubingue, profondément convaincu de l'échec du christiani-me et de toute religion, même humaniste, résolu à n'attendre le salut que de la philosophie. Celui de P. ASVELD, op. cit., est très peu religieux, comme la plupart des Aufklärem, mais il a voulu prendre plus au sérieux que ne l'ont fait ces derniers les éléments hi-toriques du christianisme et, en moderniste avant la lettre, il a insufflé à ceux-ci un sens nouveau, inspiré par l'idéalisme et le romantisme, réduisant ainsi la religion chrétienne à exprimer au plan de la Vorstellung la conscience que l'homme a de la vie infinie en lui. Très peu religieux également le jeune Hegel de A. PEPERZAK, Le jeune Hegel et la vision morale du monde, La Haye, Nijhoff, 1960 ; guidé par une image idéale de l'homme, d'inspiration grecque, inconciliable avec la conception de l'homme pécheur, le jeune Hegel aurait opté, afin de 
H.S. Harris ${ }^{9}$ des fragments de jeunesse de Hegel, que jusqu'en 1800 la religion idéale fut pour le jeune Hegel celle des Grecs, celle que, dans la Pbénoménologie, il appellera la religion de l'art ${ }^{10}$. C'est cette dernière - et non le christianisme - qui représente alors dans son esprit la religion absolue, et c'est elle qui lui sert d'étalon dans sa critique du judaïsme, de l'orthodoxie chrétienne, et, durant la période francfortoise, de la religion rationnelle des Aufklärern. Or la théologie hégélienne des oeuvres de la maturité présuppose que le christianisme soit la religion absolue, qu'en elle l'Absolu soit révélé de façon absolue. C'est. même ce présupposé que Hegel veut prendre plus au sérieux que ne le font les théologiens positifs de son temps et les Aufklärern, et ainsi développer un discours qui surmonte à la fois l'objectivisme candide des premiers et le subjectivisme des derniers, un discours qui articule l'Absolu selon la logique même de l'Absolu. On comprend dès lors que les études récentes de la Religionspbilosopbie de Hegel, intéressées par ce projet théologique et par la leçon d'audace qu'il offre à la pensée actuelle ${ }^{11}$, attendent peu de secours de l'exégèse des Jugendschriften.

maintenir cette image, pour l'athéisme. Un tout autre portrait du jeune Hegel a été présenté par T.M. KNOX, Hegel's Attitude to Kant's Etbics, in Kantstudien, 49 (1957), pp. 70-81 et par G. ROHRMOSER, op. cit. Le jeune Hegel de Knox, d'abord inspiré par l'idéal kantien de la religion naturelle, aurait redécouvert à Francfort le christianisme de l'Evangile, duquel il aurait espéré, jusqu'à sa conversion à la philosophie, la réconciliation de la raison avec la vie. Celui de Rohrmoser est un croyant, animé d'un idéal chrétien, plus précisément théologico-politique, qui he veut rien sacrifier ni du christianisme ni de la réalité politique de son temps, et qui cherche par quelle voie la religion de l'Évangile peut réussir à inspirer les structures de la société.

9. H.S. HARRIS, Hegel's Development. Toward the Sunlight 1770-1801, Oxford, Clarendon Press, 1972, 574 p. Cet ouvrage, en plus de fournir un instrument de travail indispensable à l'étude des travaux de jeunesse de Hegel, reconstruit avec plus de vraisemblance qu'on ne l'a fait jusqu'ici l'itinéraire spirituel de l'ancien séminariste du Tübinger Stift.

10. «One of the most remarkable things about the development of Hegel's philosophy is that ideas mature in a sort of steady succession and, once matured, remain fairly stable even while other ideas are developing around and above them. His conception of «reflexion» is already mature in 1800 . The conception of religion not yet mature, for Hegel's ideal model is still the religion of the Greeks, or what he called in the Pbenomenology the «religion of art». In the end this ideal is identified with «art» simply, rather than with "religion», HARRIS, op. lased., pp. 390-391.

11. «Loin de conduire à l'athéisme, une lecture complète des oeuvres de Hegel 
On ne peut pas se dissimuler cependant l'embarras dans lequel se trouve l'interprétation de la philosophie hégélienne de la religion. Car si la lecture systématique de Hegel n'a pas de peine à dévoiler dans ses oeuvres de la maturité une intentionnalité théologique, celle d'articuler la seule logique impliquée par la révélation chrétienne et de proposer la seule philosophie contenue en germe dans le message chrétien, il reste que la lecture génétique, à laquelle les chercheurs ne cessent d'avoir recours, comme pour arracher au hégélianisme un secret que l'analyse du système refuse de révéler ${ }^{12}$, aboutit presque nécessairement à montrer le jeune Hegel en train de soumettre le christianisme à une critique radicale, employant parfois, comme l'a noté Hyppolite ${ }^{13}$, des formules qui annoncent Feuerbach, et à interpréter la Religionspbilosopbie dans le sens de la thèse défendue par les jeunes hégéliens de la première génération et exposée encore récemment par K. Löwith ${ }^{14}$, selon laquelle l'intention de Hegel serait de réaliser l'Aufbebung de la religion chrétienne, d'en supprimer la forme représentative et de rendre superflu l'exercice de la foi, tout en en préservant le contenu. Il semblerait que selon la forme de lecture pratiquée, on soit tenu de faire de Hegel le dernier des grands théologiens ou le liquidateur de la foi.

Ce n'est pas mon propos ici de chercher la solution de cet embarras, ni même de mesurer les mérites respectifs des diverses méthodes de lecture appliquées à la Religionsphilosophie de Hegel. La seule question à laquelle je voudrais m'arrêter, et que les études récentes de la théologie hégélienne obligent à réexa-

invite plutôt à l'audace théologique. Loin de confondre les théologiens et de les réduire au silence, elle les convie au langage pourvu qu'il soit pétri de spéculation et non de simple érudition historique ». A. LEONARD, op. cit., pp. 393-394.

12. Dans un recueil de travaux, dont les deux premiers portent sur le rôle de Hölderlin dans la formation du système hégélien, D. HENRICH, Hegel im Kontext, Frankfurt, Surkhamp, 1967, 211 p., montre pourquoi on peut espérer de la lecture génétique de Hegel l'intelligence de son «secret». Voir notamment p. 41.

13. J. HYPPOLITE, Genèse et structure de la Phénoménologie de l'Esprit, Paris, Aubier, 1946, p. 514.

14. K. LÖWITH, Hegel's Aufbebung der cbristlicben Religion, in Hegel-Studien, 1962, pp. 193-237, reproduit dans le recueil Vorträge und Abbandlungen zur Kritik der cbristlicben Ueberlieferung, Kohlhammer, 1965, pp. 54-97. 
miner, est celle de la place de l'analyse des Jugendschriften dans la reconstruction de la philosophie hégélienne de la religion. Quel secours cette reconstruction peut-elle obtenir de leur lecture? Dans quelle mesure cette lecture peut-elle être éclairante pour comprendre le discours sur la religion que Hegel a développé dans ses oeuvres de la maturité ?

La lecture des Jugendscbriften est éclairante pour la reconstruction de la philosophie hégélienne de la religion dans la mesure où elle permet d'assister à la genèse et au développement d'une position philosophique que, dans les oeuvres de la maturité, Hegel estimera indispensable à la compréhension de la religion. Une lecture purement génétique, qui ne serait pas guidée par la pré-compréhension du système, me paraît aussi fragile qu'une lecture exclusivement systématique. Si celle-ci risque toujours d'imposer au philosophe les structures de pensée de l'interprète, celle-là évite difficilement de réduire le sens d'une philosophie à ses conditionnements historiques. Sans l'exploration des textes de jeunesse de Hegel on peut aisément se tromper sur ce que fut l'intention profonde de sa théologie, prétendre par exemple que la philosophie qu'il a développée lui parut la seule impliquée par le fait de la révélation chrétienne; sans la pré-compréhension de sa pensée achevée sur la religion, on peut tout aussi facilement adresser aux écrits du jeune Hegel des questions tout à fait étrangères à sa pensée.

Y a-t-il une thèse que Hegel, dans ses oeuvres de la maturité, estimera indispensable à la compréhension de la religion, et dont la lecture des Jugendschriften nous permet de saisir l'ébauche? Oui, et elle consiste dans la différence que l'auteur établit untre la raison et l'entendement, différence que l'Encyclopédie et les cours de Berlin rappelleront et expliciteront constamment. Abstraction faite de cette thèse, le discours de Hegel sur la religion n'est pas intelligible, il n'est même pas possible. Car si aux yeux du philosophe, la philosophie se réconcilie avec la religion, c'est à la seule condition de reconnaître les limites du Verstand et d'accéder à l'universalité de la Vernunft. La critique de l'entendement, c'est-à-dire de la forme de réflexion et de pensée abstraite qui lui est propre, constitue en vérité un moment essentiel du discours hégélien sur la religion. L'adversaire par excellence visé 
et pourchassé par Hegel, ce n'est pas la dogmatique enseignée et crue dans l'Eglise, mais la théologie de l'entendement pratiquée par les Aufklárern. Le jugement de Hegel sur cette théologie est trop connu pour que nous y insitions. Qu'il suffise de rappeler qu'elle illustre à ses yeux la vanité de l'entendement - diese Eitelkeit des Verstandes — et constitue l'adversaire le plus violent de la philosophie - die beftigste Gegnerin der Pbilosopbie - ${ }^{15}$. Avec la doctrine enseignée par l'Eglise il est possible à la philosophie de se réconcilier, car elles ont en commun le même contenu, déployé selon deux formes différentes, la représentation et le concept; mais son désaccord avec la théologie de l'entendement est irrémédiable du fait qu'elles n'ont rien en commun, ni la forme, ni le contenu. Cette théologie, qui a toujours à la bouche le mot «raison», et se présente toujours comme l'oeuvre de la raison, alors qu'elle n'est en fait que le produit d'un entendement désséché, vide en réalité la religion de son contenu, car elle réduit Dieu à un universel abstrait, à un infini opposé au fini, à un être suprême indéterminé dont on ne connaît rien $^{16}$. C'est, soutient Hegel, la paradoxe de cette théologie de

15. «Die Aufklärung, diese Eitelkeit des Verstandes, its die heftigste Gegnerin der Philosophie; sie nimmt es übel, wenn diese die Vernunft in der christlichen Religion aufzeigt, wenn sie zeigt, dass das Zeugnis de; Geistes, der Wahtheit in der Religion niedergelegt ist. In der Philosophie, welche Theologie ist, ist es einzig darum zu tun, die Vernunft der Religion zu zeigen ». Vorlesungen über die Pbilosophie der Religion, Theorie Werkausgabe, Suhrkamp, 1969, vol. 17. p. 341.

16. "Die Philosophie ist dagegen dem sogenannten Rationalismus in der neueren Theologie entgegen; dieser hat die Vernunft immer im Munde, es ist aber nur trokener Verstand. Von der Vernunft ist nichts darin zu erkennen als das Momenc des Selbstdenkens; es ist aber nur abstraktes Denken. Der Rationalismus ist der Philosophie dem Inhalt und der Form nach entgegengesezt; er hat den Inhalt, hat den Himmel leer gemacht, alles zu endlichen Verhaltnissen heruntergesetzt. Und auch der Form nach ist $\mathrm{cr}$ der Philosophie entgegengesetzt, denn seine Form ist Räsonieren, unfreies Räsonieren, nicht Begreifen. Der Supranaturalismus ist in der Religion dem Rationalismus ent. gegengesetzt, aber er ist der Philosophie in Ancehung des wahrhaften Inhalts verwandt, aber der Form nach verschieden; denn er ist ganz geistlos, hölzern gekorden und nimmt äusserliche. Autorität zur Rechtfertigung an. (...) Die Philosophie als begreifendes Denken dieses Inhaits hat in Rücksicht auf das Vorstellen der Religion den Vorteil, dass sie beides versteht; sie kann die Religion verstehen, sie versteht auch den Rationalismus und den Supranaturalismus, und auch sich; aber nicht ist es auch umgekehrt der Fall 》. Vorlesungen über die Geschichte der Pbilosophie, Theorie Werkausgabe, vol. 18 , p. 101. 
l'Aufklarung d'avoir voulu rationaliser le christianisme, le soustraire à l'autorité et n'en retenir que ce que la raison y découvre, et d'avoir en même temps combattu la raison en soutenant qu'il lui est impossible de connaître la nature de Dieu ${ }^{17}$. Mais c'est le résultat nécessaire auquel aboutit la raison, lorsqu'elle se comporte comme entendement, se donnant pour règle ultime l'identité abstraite : pour elle Dieu ne peut être que l'Un, l'Etre des êtres, l'Identité vide sans différenciation ${ }^{18}$. Elle est impuissante à surmonter l'opposition entre le fini et l'infini, le particulier et l'universel, le concret et l'abstrait, et à reconnaître que la vérité est quelque chose de concret, d'infini dans lequel le fini est contenu, d'universel posant ses propres déterminations, ou, en d'autres termes, à penser que Dieu est Esprit.

Le contenu de la religion, qui est un mystère pour le Verstand ${ }^{19}$, n'est accessible qu'à la Vernunft. Ce contenu c'est

17. «Dagegen ist es andererseits ebenso bekannt, dass die sogenannte Vernunft sich geltend gemacht, den Glauben aus Autorität verworfen, das Christentum vernünftig gemacht hat, so dass die eigene Eincicht, die eigene Ueberzeugung durchaus nur verpflichtend für mich sei, etwas anzuerkennen. Aber wunderbarweice ist diese Behauptung des Rechts der Vernunft dahin umgeschlagen, dies zum Resultate zu haben, dass die Vernunft nichts Wahres erkennen könne. Diese sogenannte Vernunft bekämpfte einerseits den religiösen Glauben im Namen und kraft der denkenden Vernunft, - und zugleich ist sie ebenso gegen die Vernunft gekehrt, Feindin der Vernunft, behauptet gegen sie die innere Ahnung, das Gefühl, macht so das Subjektive zum Masstabe des Geitenden, - eine eigene Ueberzeugung, wie jeder sie in seiner Subjektivität sich aus und in sich selber mache ». Ibid., p. 31.

18. «Denn das Prinzip des Verstandes ist die abstrakte Identität mit sich, nicht die konkrete, dass diese Unterchiede in einem sind. Für den Verstand ist Gott das Eine, das Wesen der Wesen. Diese unterschiedslose, leere Identität ist das falsche Gebild des Verstandes und der modernen. Theologie. Gott ist Geist, das sich Gegenständlichmachende und sich darin selbst wissend, d.i. die konkrete Identität, und so ist die Idee auch ein wesentliches Moment. Aber nach der abstrakten Identität sind das eine und das andere selbständig für sich, und ebenso beziechen sie sich aufeinander: also ist der Widerspruch da. 》Vorlesungen über die Pbilosopbie der Religion, vol. 17, p. 230.

19. «Diese spekulative Idee ist dem Sinnlichen entgegengesetzt, auch dem Verstande; sie ist daher ein Gebeimnis für die sinnliche Betrachtungsweise und auch für den Verstand. Für beide ist sie ein «mysterion 》d.h. in Absicht auf das, was das Vernünftige darin ist. Ein Geheimnis im gewöhnlichen Sinn ist die Natur Gottes nicht, in der christlichen Religion am wenigsten; da hat sich Gott zu erkennen gegeben, gezeigt was er ist, da ist er offenbar. Aber ein Geheimnis ist es für das sinnliche Wahrnehmen, Vorstellen, für die sinnliche Betrachtungsweise und für den Verstand 》. Vorlesungen über die Pbilosopbie der Religion, vol. 17, p. 227 . "Wir haben die Idee rein spekulativ zu 
l'identité concrète de l'universel qui se particularise, de l'infini qui s'autodétermine, de la Raison qui est en soi et pour soi ${ }^{20}$. Or ce contenu ne trouve son élément propre que dans la raison, à condition que celle-ci se reconnaisse comme raison universelle, comme ce qu'il y a de divin chez l'homme, comme Esprit de Dieu ${ }^{21}$. Dans la mesure où l'homme renonce à sa particularité, se comporte comme conscience universelle et laisse dominer en lui le penser rationnel, dans la même mesure le contenu de la religion reçoit la forme qui lui convient ${ }^{22}$. Le penser de ce contenu, par une raison qui se comporte comme raison et non comme entendement, déploie une théologie tout à fait différente de celle qu'on lui a enseignée au Tübinger Stift et dont il a vraisemblablement conservé un souvenir amer. Celle-ci, qu'elle se présente comme räsonierende Exegese de l'Ecriture ou comme Verstandesmetaphysik, s'appuie toujours sur un raisonnement particulier et arbitraire, de plus impuissant à critiquer ses propres présupposés. La Religionsphilosophie, parce qu'elle est l'articulation de la raison

betrachten und sie gegen den Verstand zu rechtfertigen, gegen ihn, der sich gegen allen Inhalt der Religion überhaupt empört. Dieser Inhalt heisst Mysterium, weil er dem Verstande ein Verborgenes ist, denn er kommt nicht zu dem Prozess, der diese Einheit ist: daher ist alles Spekulative dem Verstande ein Mysterium». Ibid,, p. 535. «Die Mysterien sind ihrer Natur nach, als spckulativer Inhalt, geheim für den Verstand, nicht für die Vernunft; sie sind getade das Vernünftige in Sinne des Spekulativen. Der Verstand fasst das Spekulative nicht, dies Konkrete; der Verstand hält die Unterschiede schlechthin getrennt fest. Ihren Widerspruch enthält das Mysterium auch, es ist aber zugleich auch die Auflösung desselben. 》Vorlesungen über die Gescbicbte der Pbilosopbie, vol. 18, p. 100-101.

20. «Die Philosophie steht mit der Religion auf gleichem Boden, hat denselben Gegenstand: die allgemeine, an und für sich seiende Vernunft; der Geist will sich diesen Gegenstand zu eigen machen, wie in der Religion es in der Andacht und dem Kultus geschiet. 》Vorlesungen über die Geschichte der Pbilosopbie, vol. 18, p. 83.

21. «Die menschliche Vernunft, das Bewusstsein Wesens, ist Vernunft überhaupt, das Göttliche im Menschen; und der Geist, insofern er Geist Gottes ist, ist nicht ein Geist jenseits der Sterne, jenseits der Welt, sondern Gott ist gegenwärtig, allgegenwärtig und als Geist in allen Geistern. 》 Vorlesungen über die Pbilosophie der Religion, vol. 16, p. 40.

22. «Je mehr der Mensch im vernünftigen Denken die Sache selbst in sich walten lässt, auf seine Partikularität Verzicht leistet, sich ais allgemeines Bewusstsein verhält, seine Vernunft nicht das Ihrige im Sinne eines Besonderen sucht, um so weniger wird sie in jenen Gegensatz fallen; denn sie, die Vernunft, ist selbst die Sache, der Geist, der göttliche Geist. 》Ibid., p. 41. 
universelle, démasque l'arbitraire de cette entreprise ${ }^{2 \mathbf{3}}$, en même temps qu'elle se présente comme la conscience que Dieu a de soi, ou mieux, la conscience de soi qui se sait dans le savoir humain ${ }^{24}$. Parce que cette théologie spéculative est celle d'une raison qui se sait être l'être — die Vernunft ist selbst die Sache der Geist, der göttliche Geist - et qui se retrouve dans la religion, elle réalise l'authentique pensée libre, car être libre, c'est ne pas se rapporter à une altérité, ne pas dépendre de quelque chose d'autre ${ }^{25}$.

Cette thèse sur la différence entre la Vernunft et le Verstand, dont le rôle capital dans la philosophie définitive de Hegel ne peut être ici que rappelé, s'est imposée très tôt à l'esprit de ce dernier, bien avant qu'il eut élaboré une philosophie de la religion. Il l'avait clairement formulée et avait compris les implications qu'elle exigeait pour l'étude philosophique de la religion, avant d'avoir rédigé la Phénoménologie. Ainsi lorsqu'on consulte les premières publications d'Iéna, l'écrit sur La différence entre les systèmes de Fichte et de Schelling, les articles rédigés en 1802-1803 pour le Kritisches Journal der Philosopbie, on n'a pas de peine à observer qu'au moment de son entrée dans le

23. «In der Weise wie jene Verstandesmetaphysik und die räsonierende Exegese kann daher die Religionsphilosophie der Positiven Religion und der Lehre der Kirche, die ihren Inhalt noch bewahrt hat, nicht entgegenstehen. Es wird sich vielmeher zeigen, dass sie der positiven Lehre unendlich näher steht, als auf den ersten Anblick scheint, ja das die Wiederherstellung der vom Verstande auf das Minimum reduzierten Kirchenlehre so sehr ihr Werk ist, dars sie gerade um dieses ihres wahrhaften Inhalts wegen von der nut verständigen Vernunfttheologie als Verdüsterung des Ge1stes verschrien wird. Die Angt des Verstandes und sein Hass gegen die Philosophie kornmt aus der Besorgnis her, das er sieht, sie führe sein Reflekticren auf den Grund zurück, d.h. zum Affirmativen, worin es zugrunde geht, und komme doch zu einem Inhalt, zu einer Erkenntnis der Natur Gottes, nachdem aller Inhalt bereits aufgehoben zu sein schien. Ibid., p. 39-40.

24. *...es ist das Nähere darin enthalten, dass nicht die sogenannte menschliche Vernunft und ihre Schranke es ist, welche Gott erkennt, sondern der Geist Gottes im Menscben; e; ist, nach dem vorhin angeführten spekulativen Ausdruck, Gottes Selbstbewusstsein, welches sich in dem Wissen des Menschen weis; 》. Ibid., vol. 17, p. 385.

25. «Nur dies ist Freiheit; frei ist, was nicht auf ein Anderes sich bezieht, nicht vom ihm abhängig ist. Der Geist, indem er zu sich selbst kommt, erreicht dies, (ein) freier zu sein. "Vorlesungen übor die Geschichte de Pbilosophie, vol. 18, p. 42. 
monde universitaire, Hegel se servait de cette thèse comme d'un principe auquel il avait constamment recours pour effectuer sa critique des philosophies de son temps. C'est elle qui lui permettait de juger les entreprises de son époque, d'y détecter ce qu'il y avait de non-philosophique, voire de définir la tâche de la philosophie authentique. La philosophie qu'il estime inauthentique, c'est celle qui ravale la Vernunft au Verstand et qui, tout en se présentant comme le discours de la raison, se comporte en réalité comme un entendement. Les systèmes de Kant et de Fichte, qui ont pourtant fourni à la pensée philosophique le principe de la spéculation, ne dépassent pas encore le niveau d'une Vernunft réduite à un Verstand ${ }^{26}$. Comment Hegel comprend-il cette différence? L'entendement, c'est le pouvoir de limitation et d'opposition; il s'épuise à transformer et à fixer l'absolu en concepts opposés, et donc finis, à consommer la scission entre la conscience et la totalité. «Plus la construction de l'entendement est solide et brillante, plus la vie, qui y est emprisonnée comme une partie, devient inquiète, et s'efforce de s'en évader pour pénétrer dans la liberté ${ }^{27}$. La fonction de la Vernunft est de supprimer ces oppositions. Elle est la manifestation de l'Absolu, sa force négative; dans la mesure où elle se développe comme relation à l'Absolu, elle anéantit les produits

26. Voir dans l'avant-propos du Differenzscbift le jugement de Hegel sur Kant : "In jener Deduktion der Vertandesformen ist das Prinzip der Spekulation, die Identität des Subjekts und Objekts, auf bestimmteste ausgesprochen; diese Theorie des Verstandes ist von der Vernunft über die Taufe gehalten worden. - Hingegen wenn nun Kant diese Identität sebst, als Vernunft, zum Gegenstand der philosophischen Reflexion macht, verschwindet die Identität bei sich selbst; wenn der Verstand mit Vernunft behandelt worden war, wird dagegen die Vernunft mit Verstand behandelt. 》 et sur Fitche : "Sie (die Spekulation) übergibt die Vernunft dem Verstand und geht in die Kette der Endlichkeiten des Bewusstseins über, aus welchen sie sich zur Identität und zur wahren Unendlichkeit nicht wieder rekọnstruiert. » Differenz des Fichtes'scben und Scbellings'schen Systems, in Jenaer Kritische Schriften, éd. H. BUCHNER-O. POGGELER, p. 6.

27. «Dic Vernunft erreicht das Absolute nur, indem sie aus diesem mannigfaltigen Teilwesen heraustritt; je fester und glänzender das Gebäude des Verstandes ist, desto unruhiger wird das Bestreben des Lebens, das in ihm als Teil befangen ist, aus ihm sich heraus in die Freiheit zu ziehen $»$. Ibid., p. 12-13. 
fixes et limités de l'entendement et leur accorde ainsi la seule consistance qu'on peut leur octroyer ${ }^{28}$.

Dans l'important article de 1802, Glauben und Wissen, la différence entre le Verstand et la Vernunft sert à Hegel de clé pour juger les philosophies religieuses de l'Aufklärung et pour définir la seule façon légitime selon laquelle la philosophie peut se rapporter à la religion. On y trouve ébauchés et la critique de la Verstandesmetaphysik que Hegel a développée dans ses oeuvres ultérieures et le programme d'une Religionspbilosopbie qui sera l'oeuvre de la Vernunft. Le tort des Aufklarern, soutient-il, a été à la fois de rabaisser la raison à l'entendement et de déformer la religion. " $\mathrm{Ni}$ la religion positive contre laquelle l'Aufklárung partit en guerre ne demeura religion, ni la raison victorieuse, ne demeura raison, et le rejeton, qui plane triomphalement sur ce cadavre, qu'il unit à titre d'enfant de la paix, tient aussi peu de la raison que l'authentique foi ${ }^{29}$. Cette impuissance de la raison à se comporter comme raison et son incapacité à comprendre le vrai sens de la religion représentent, à ses yeux, deux phénomènes indissociablement unis. Ce qui entraîna le rabaissement de la raison à l'entendement, chez les Aufklärern, c'est leur incapacité de comprendre la religion de façon idéaliste. "Déjà déchue en soi et pour soi, pour avoir compris la religion seulement comme quelque chose de positif, et non de façon idéaliste, la raison ne put désormais, après la bataille, rien faire de mieux que de se regarder, de parvenir à la connaissance de soi et de reconnaittre son néant; car le meilleur d'elle-même, puisqu'elle n'est qu'entendement, elle le pose en dehors et au-dessus d'elle dans la foi; ce fut le cas pour les philosophies de Kant, de Jacobi et de Fichte; ainsi se fait-elle de nouveau servante d'une foi ${ }^{30}$. Le meilleur d'elle-même,

28. «Indem es als Vernunft in die Ferne tritt, ist die Totalität der Beschränkungen zugleich vernichtet, in diesem Vernichten auf das Absolute bezogen und hiermit als blosse Erscheinung begriffen und gesetzt; die Entzweiung zwischen dem Aboluten und der Totalität der Berschränkungen ist verschwunden. »Ibid., p. 13.

29. Glauben und Wissen in Jenaer Kritische, Scbriften éd. BUCHNER-POGGELER, p. 315. J'emprunte la traduction de M. MERY, G.W.F. Hegel. Premières publications, Paris, Vrin, 1952, p. 193.

30. "Die Vernunft, welche dadurch an und für sich schon herunter gekommen war, dass sie die Religion nur als etwas Positives, nicht idealistisch auffaste, 
que la raison de l'Aufklârung, se comprenant comme entendement, pose en dehors de soi, c'est l'Absolu. «Ce fait, qui autrefois passait pour la mort de la philosophie, c'est-à-dire que la raison dût renoncer à son existence dans l'Absolu, et partant s'en exclure complètement et ne se comporter que négativement à son égard, devint désormais le sommet de la philosophie et le néant de l'Aufklärung en devenant conscient a été érigé en système ${ }^{31}$.

Dans ces textes d'Iéna, Hegel proclame une conviction dont s'inspirera sa Religionspbilosopbie. Pour être possible, celle-ci doit comprendre la religion, non pas seulement comme une réalité positive, mais dans son idéalité, et quiconque ne la comprend pas de cette façon renonce par le fait même à la raison et s'enferme dans les limites de l'entendement. Or cette conviction présuppose que Hegel, avant d'arriver à Iéna, fût déjà parvenu à découvrir le sens idéaliste de la religion et à saisir la fonction authentique de la raison, dans sa différence avec l'entendement. Il s'ensuit que pour comprendre la genèse de cette conviction de base et le rôle qu'elle a joué dans le développement de la pensée hégélienne, l'interprète de la Religionspbilosophie doit entreprendre l'analyse des écrits antérieurs à 1801.

La lecture des Jugendschriften, refaite à ce point de vue, prend un intérêt étonnant. Elle révèle, en premier lieu, que la différence entre la Vernunft et le le Verstand constitua pour le jeune Hegel l'une des positions les plus fondamentales de sa pensée, une sorte d'inaliénable auquel s'appuie l'organisation de son discours. Elle montre également que c'est en méditant cette

hat nichts besseres tun können, als nach dem Kampf nunmehr auf sich zu sehen, zu ihrer Selbstkenntnis zu gelangen und ihs Nichtsein dadurch anzuerkennen, dass sie das Bessere, als sie ist, da sie nur Verstand ist, als ein Jeseits in einem Glauben ausser und über sich setzt, wie in den Pbilosopbien Kants, Jacobis und Ficbtes geschehen ist, und dass sie sich wieder zur Magd eines Glaubens macht. 》Ibid., pp. 315-316. Je corrige ici la traduction de MERY qui me paraît fautive.

31. «... und was sonst für den Tod der Philosophie galt, dass sie die Vernunft auf ihr Sein im Absoluten Verzicht tun sollte, sich schlechthin daraus anschlösse und nur negativ dagegen verhielte, wurde nunmehr der höchste Punkt der Philosophie, und das Nichtsein der Aufklärung ist durch das Bewusstwerden über dasselbe zum System geworden. I Ibid., p. 316. 
différence, et en obtenant de la sorte une nouvelle compréhension de la Vernunft, qu'il fut conduit à rompre avec le kantisme et avec la théologie «rationnelle» de l'Aufklarung pour adopter une interprétation idéaliste de la religion.

La différence entre le Vernunft et le Verstand apparaît pour la première fois sous la plume de Hegel dans deux documents de ses années de gymnase à Stuttgart. Le premier est une transcription faite en mars 1787 d'un passage extrait de l'ouvrage de Garve sur l'Examen des Facultés. L'intérêt de ce texte, sur lequel Lacorte et Harris ${ }^{32}$ ont attiré l'attention, vient du fait que pour formuler l'opposition Vernunft-Verstand, le jeune étudiant modifie le texte qu'il transcrit. "Des matériaux fournis par la sensibilité et l'imagination, écrit-il transcrivant le texte de Garve, la raison construit le système de concepts généraux selon lesquels l'homme se règle lui-même et son agir. La raison abstrait; et si cela se répète plusieurs fois, elle prend le nom de réflexion; et puisqu'avant même que l'âme soit capable d'abstraire, le langage lui fournit des concepts abstraits, liés aux mots, l'entendement [Verstand, alors qu'ici Garve avait écrit Vermunft] soccupe avant tout de déterminer le sens des mots et de chercher l'idée générale dont le mot doit être le signe ${ }^{33}$. En transcrivant ce texte, dont il modifie un mot important, Hegel cherche à exprimer la différence entre deux formes de connaissance: l'une réservée à la Vernunft, est tirée de l'expérience et vise à formuler un système de pensée régulateur de la vie, l'autre, propre au Verstand, est reçue par la médiation du langage. Au savoir livresque du Verstand, il oppose donc la connaissance personnelle, oeuvre de la Vernunft, tirée du fonds de l'expérience et ordonnée à la direction de l'action.

Dans une dissertation rédigée l'année suivante (1788), Hegel se sert du même contraste entre ces deux démarches intellectuelles, pour comparer entre elles la culture des anciens et celle des modernes. Chez les anciens, il admire deux qualités qu'il ne retrouve pas chez les modernes: leur simplicité et leur

32. C. LACORTE, $I$ primo Hegel, Firenze, Sansoni, 1959, p. 94 ; H.S. HARRIS, op. cit., D. 36-37.

33. Dokumenie zu Hegel's Entwicklung, berausgegeben von Jobannes Hoffmeister, Stuttgart, Fromman, 1936, p. 122. 
originalité. Les poètes anciens savaient exprimer les choses avec la plus grande simplicité ; ils savaient traduire chaque sentiment «sans y distinguer le multiple, comme peut le faire l'entendement ${ }^{34}$. En outre leur pensée était le fruit d'une expérience personnelle. «Le système entier d'éducation et de culture des anciens fut ainsi que chacun acquérait ses propres idées à partir de son expérience; on ne connaissait pas la froide érudition livresque qui imprime dans le cerveau par des signes morts; chacun devait être original. Nous, au contraire, dès notre enfance, apprenons une masse de mots et de signes d'idées qui reste dans nos têtes, inactive et inutilisée; seulement petit à petit arrivons-nous, par l'expérience, à connaître le trésor que nous possédons et à penser quelque chose à l'aide des mots ${ }^{35}$.

De ces premiers documents, que nous aurions tort de trop presser, il ressort qu'avant même de connaître la philosophie pratique de Kant, le jeune Hegel entretenait un idéal de raison inspiré, semble-t-il, par sa lecture des anciens. Cet idéal, il l'exprimait par le contraste de la Vernunft et du Verstand, pour souligner ce que la raison n'est pas. Dans ce contraste, la raison est comprise par le jeune Hegel comme n'étant réductible ni à une faculté de connaissances venues de l'extérieur, communiquées par voie d'enseignement, ni à un pouvoir d'analyse qui décompose l'unité du tissu expérientiel déjà harmonieusement organisé. En elle il voit, au contraire, la source d'idées issues de l'expérience, acquises par voie de découverte personnelle et ordonnées à articuler et à réaliser l'unité de l'expérience. C'est cet idéal d'une raison autonome, principe d'unité de la vie humaine,

4. Dokumente, p. 49. - Six ans plus tard, Schiller, dans ses Briefe über die aestbetische Erziebung, caractérisera aussi par sa Simplizität l'humanisme grec: "Die Griechen beschämen uns nicht bloss durch eine Simplizität, die unsern Zeitalter fremd ist; sie sind zugleich unsere Nebenbuhler, ja oft unsere Muster in den nämlichen Verzügen, mit denen wir uns über die Naturwidrigkeit unserer Sitten zu tröster pflegen》 (Lettre VI), édition bilingue de R. LEROUX, Paris, Aubier, 1943, p. 102. - A noter également que, dans une note ajoutée à la Lettre XVIII, Schiller oppose la Vernunft au Verstand en réservant à la première la réalisation de l'unité dans la réalité humaine: «Die Natur (der Sinn) vereinigt überall, der Verstand scheidet überall, aber die Vernunft vereinigt wieder; daher ist der Mensch, ehe er anfängt zu philosophieren, der Wahrheit näher als der Philosoph, der seine Untersuchung noch nicht geendigt hat $\gg$. Ibid., p. 234. 
qui ne cessera de révéler ses implications au jeune Hegel durant les dix prochaines années.

On a trop peu remarqué, à mon avis, le rôle exercé par cette thèse de la différence entre la Vernunft et le Verstand dans les premiers travaux de critique de la religion qui occupèrent le jeune Hegel durant les derniets mois de résidence au Tübinger Stift et durant son séjour à Berne (1793-1796). Dans cette critique, il est vrai, Hegel se présente comme kantien résolu. Son essai de Tubingue sur la Volksreligion ${ }^{36}$, ses fragments de Berne sur $L a$ positivité de la religion chrétienne ${ }^{37}$, ses ébauches d'une critique du christianisme ${ }^{38}$, son curieux Leben Jesu ${ }^{39}$, le montrent occupé à poursuivre jusque dans ses ultimes conséquences la perspective ouverte par Kant sur la religion. Le kantisme, dont il attend «une révolution en Allemagne ${ }^{40}$ et à l'égard de laquelle il ne veut pas rester inactif, consiste surtout, pour le jeune Hegel de cette époque, dans la thèse sur la religion que développent la deuxième Critique et l'essai sur La religion dans les limites de la raison. La religion a sa source dans un besoin de la raison pratique et est essentieliement ordonnée à la réalisation de la moralité de l'homme. Cette idée kantienne dont il fait le principe de sa critique de l'orthodoxie, le jeune Hegel veut en déployer toutes les implications, tout comme, quarante ans plus tard, les jeunes hégéliens de la première génération chercheront à montrer les conséquences du hégélianisme en matière de religion. Polémiquer contre la théologie existante, empêcher que ce que Kant a produit soit déformé et affadi par les théologiens «qui se servent des matériaux critiques pour consolider leur temple gothique ${ }^{41}$, ébranler l'orthodoxie, tel est, si l'on se fie à sa correspondance avec Schelling, le programme du jeune précepteur de Berne.

35. Dokumente, p. 49-50.

36. NOHL, H., Hegel's theologische Jugendschriften, Tübingen, 1907. pp. 3-39.

37. Ibid, pp. 152-213.

38. Ibid., pp. 48-50.

39. Ibid., pp. 73-136.

40. Lettre de Hegel à Schelling, 16 avril 1795, in Briefe von und an Hegel, éd. J. HOFFMEISTER, I, p. 23.

41. Lettre de Hegel à Schelling, fin janvier 1795, Ibid., pp. 16-17. 
Il importe toutefois, si l'on veut comprendre le kantisme du jeune Hegel, de reconnaître qu'il ne fut pas le point de départ de son itinéraire spirituel. Si la philosophie religieuse de Kant trouva chez lui un accueil si enthousiaste, c'est que déjà il s'était formé un idéal de rationalité, que la religion, à ses yeux, devait servir à réaliser dans le peuple. C'est dans cet idéal qu'il faut voir, me semble-t-il, l'inaliénable, constamment présupposé par le développement ultérieur de sa philosophie. Si l'on a dit du cartésianisme qu'il eut sa source dans le plaisir que prit le jeune Descartes aux classes de mathématiques, ne peut-on pas dire du hégélianisme qu'il a été le fruit d'un idéal de raison, que le jeune Hegel embrassa dès les années de gymnase, que la critique de la religion des Jugendscbriften lui permit d'articuler et dans lequel il a vu, dès le début de son séjour à Iéna, la possibilité de comprendre la religion positive, en retenant intégralement son contenu sans rien sacrifier des exigences de la raison?

Plus profondément que l'idée kantienne de religion, c'est le contraste entre le Vernunft et le Verstand qui, dans les travaux de Tubingue et de Berne, préside à l'effort déployé par le jeune Hegel pour définir les conditions selon lesquelles une religion objective et positive peut devenir subjective et morale. La religion objective, pour lui, est l'oeuvre de l'entendement et de la mémoire. Faite de connaissances qui reposent dans la tête comme un capital mort et qui se laissent ordonner en systèmes, elle n'est plus une religion, mais seulement une théologie. La religion subjective, au contraire, est affaire du coeur, elle répond au besoin de la raison de pénétrer de rationalité le tissu entier de la nature humaine et se traduit par le sentiment et l'action ${ }^{42}$.

42. «Objektive Religion ist fides quae creditur, der Verstand und das Gedächtnis sind die Kräfte, die dabei wirken, die Kenntnisse erforschen durchdenken und behalten oder auch glauben. Zur objektiver Religion können auch praktische Kenntnisse gehören, aber insofern sind sie nur ein totes Kapital - die objective Religion lässt sich im Kopfe ordnen, sie lässt sich in ein System bringen, in einem Buche darstellen und andern durch die Rede vortragen; die subjektive Religion äussert sich nur in Empfindungen und Handlungen ». NOHL, p. 6. - «Wenn ich von Religion spreche, so abstrahiere ich schlechterdings von aller wissenschaftlichen oder vielmeher metaphysischen Erkenntnis Gottes, unseres und der ganzen Welt Verhältnisses zu ihm usw. Eine solche Erkenntnis, bei der sich bloss der räsonierende Verstand beschäftigt, ist Theologie, nicht mehr Religion. Ich 
L'insistance du jeune Hegel pour voir à l'oeuvre dans ces deux types de religion deux pouvoirs différents de l'âme, l'entendement et la raison, et pour discréditer le rôle du Verstand dans la religion révèle son attachement à l'idée de raison, telle qu'elle s'était imposée à lui dès ses années de collège. «La religion, écrit-il, gagne très peu de l'entendement, dont les opérations et les doutes refroidissent le coeur plus qu'ils ne le réchauffent ${ }^{43}$, «il ( l'entendement) est comparable à un courtisan qui se dirige d'après l'humeur de son maitre, capable de justifier chaque passion et chaque entreprise, toujours au service de l'amour propre $^{44}$; «éclairer l'entendement ne rend pas l'homme meilleur, il le rend seulement plus prudent ${ }^{45}$. La religion que cherche le jeune Hegel ne sera pas une théologie de l'entendement; elle devra être en harmonie avec son idéal de raison : ce pouvoir que nous avons d'être les auteurs de toute législation et de nous retrouver en tout ce qui émane de la raison. Elle devra être la noutrice qui aide chacun à construire sa petite maison, que chacun peut reconnaitre comme sienne ${ }^{46}$.

Il suffit de lire la conclusion ajoutée par Hegel, le 29 avril 1796, à son essai sur La positivité de la religion chrétienne, pour comprendre le rôle qu'il accorde à la différence entre Vernunft et Verstand et le sens qu'il lui donne pour sa critique de l'orthodoxie. "L'erreur fondamentale de tout le système d'une église, écrit-il, consiste dans sa méconnaissance des droits de chaque puissance de l'esprit humain, et surtout de la première entre toutes, la raison (...) La séparation salutaire, effectuée par Kant pour la science, entre les domaines respectifs des pouvoirs

rechne hier nur insoweit Kenntnisse von Gott und Unsterblichkeit zur religion, als das Bedürfniss der praktischen Vernunft fordert und was in einem leicht einzusehenden Zusammenhang damit steht». Ibid., p. 8. «Wenn Theologie Sache des Verstandes und des Gedächtnisses ist - ihr Ursprung mag übrigens sein, woher er will, aus der Religion selbst - , Religion aber Sache des Herzens, wegen einer Bedürfnisses der praktischen Vernunft interessant, so erhellt von selbst dass verschiedene Seelenkräfte bei Religion und Theologie wirksam (sind) und auch verschiedene Vorbereitungen des Gemüts für beide erfordert werden ». Ibid., p. 9.

43. Ibid., p. 10.

44. Ibid., p. 12.

45. Ibid., p. 12.

46. Ibid., p. 17. 
de l'esprit, n'a pas été faite par la législation de l'Eglise, et il se passera encore des siècles avant que l'esprit des Européens, dans la vie quotidienne et dans la législation, comprenne et fasse connaitre cette distinction, à laquelle pourtant les Grecs, grâce à la justesse de leur sentiment, sont parvenus d'eux-mêmes (worauf das richtige Gefühl der Griechen sie von selbst gebracht hatte). Dans la religion grecque, et en toute religion dont le principe est la pure morale, les préceptes de la raison ne sont pas traités comme des règles de l'entendement; ceux-là sont subjectifs, tandis que celles-ci sont objectives. L'Eglise chrétienne au contraire, expose le subjectif de la raison comme quelque chose d'objectif, comme règle ${ }^{47}$. Ce qui ne pourra être réalisé qu'après une longue éducation de l'esprit européen, et que le christianisme aurait méconnu, c'est, pour Hegel, l'écart entre Ja Vernunft et le Verstand, écart que Kant a été le premier à formuler conceptuellement ${ }^{48}$, mais qui se trouvait déjà vécu par les Grecs. En quoi consiste-t-il? Se sentant sans doute plus près des Grecs que de Kant qui n'a jamais jugé nécessaire pour mettre en lumière l'autonomie de la raison de discréditer l'entendement, Hegel oppose, comme il le fait dans ses travaux d'étudiant à Stuttgart, la subjectivité de la raison à l'objectivité de l'entendement. La fonction de la raison, ce premier de tous les

47. «Der Grundefehler, der bei dem ganzem System einer Kirche zum Grunde liegt, ist die Verkennung der Rechte einer jeden Fähigkeit des menschlichen Geistes, besonders der ersten unter ihnen, der Vernunft; und wenn diese durch das System der Kirche verkennt worden ist, so kann das System der Kirche nicht anderes als ein System der Verachtung der Menschen sein. Die heilsame Trennung des Gebiets der Kräfte des menschlichen Geistes, die Kant für die Wissenschaft gemacht hat, diese Trennung ist von der Gesetztgebung der Kirche nicht gemacht worden, und Jahthunderte werden noch vergehen, bis der Geist der Europäer im tätigen Leben, in den Gesetztgebungen jenen Unterschied wird $\epsilon$ rkennen und machen lernen, worauf das richtige Gefühl der Griechen sie von selbst gemacht hatte. Die moralischen Gebote der Vernunft werden nämlich «in der Religion der Griechen» so wie in jeder, deren Prinzip reine Moral ist, «nicht» wie Regeln des Verstandes behandelt und aufgestelit, jene sind subjektiv, diese objektiv; von der christlichen Kirche, hingegen wird das Subjektive der Vernunft wie etwas Objektives als Regel aufgestellt ». Ibid., p. 211. J'adopte les modifications du texte manuscrit proposées par NOHL et indiquées par les guillemets.

48. C'est toujours à Kant que, dans ses oeuvres de la maturité, Hegel attribuera la paternité de la différence entre Vernunft et Verstand. Voir par exemple le Zusatz du \#467 de l'Encyclopédie. 
pouvoirs dont est doté l'esprit, est de donner à l'homme le droit inaliénable de se constituer sa propre loi, sich selbst sein Gesetz $z u$ geben, sich selbst Gesetzgeber zu sein. Ce n'est pas l'universalité de cette loi, ni sa nécessité, qui intéressent d'abord le jeune Hegel exclusivement absorbé à cette époque par l'éducation populaire. Ce qui le préoccupe, c'est de définir les conditions concrètes qu'il faut réaliser pour permettre à cet idéal de raison de renaître dans la conscience européenne et de se manifester au monde.

Dans les textes de la période francfortoise (1797-1800), on ne retrouve plus ce contraste entre Vernunft-Verstand. Il ne sert plus à exprimer les deux démarches spirituelles auxquelles correspondaient, dans la période précédente, deux formes opposées de religion. Ni les fragments sur L'amour et la religion ${ }^{49}$, ni le groupe d'essais sur L'esprit du cbristianisme et son destin ${ }^{50}$, ni la nouvelle introduction à l'essai sur La Positivité ${ }^{51}$, ni le célèbre Systemfragment de $1800^{52}$, ne le conservent. De la Vernunft, il est d'ailleurs peu question dans ces écrits, et lorsqu'Hegel en parle, c'est pour la mettre sur le même pied que l'entendement: Vernunft et Verstand désignent tous deux le pouvoir de réflexion ou de conceptualisation ${ }^{53}$. Ce n'est plus l'en-

49. NOHL, pp. 374-382.

50. Ibid., pp. 261-342.

51. Ibid., pp. 139-151.

52. Ibid., pp. 345-351.

53. Voir à titre d'exemple l'introduction de 1800 à l'essai sur La positivité. «Schon aus dieser Entgegensetzung erhellt, dass eine positive Religion eine wider-oder- übernatürliche wäre, welche Begriffe, Kenntnisse enthält, die für den Verstand und die Vernunft überschvenglich sind... 》 NOHL, p. 140. «Nur wenn das Ueberflüssige die Freiheit aufhebt, wird est positiv, d.h. wenn es Prätention gegen den Verstand und die Vernunft macht und deren notwendigen Gesetzen widerspricht. Die Allgemeinheit dieses Kriterium muss dadurch geschränkt werden, dass Verstand und Vernunft nur dann Richter sein können, wenn an sie appeliert wird; was keinen Anspruch darauf macht, verstäding oder vernünfting zu sein, gehört durchaus nicht in ihre Gerichtsbarkeit. Und hierin liegt ein Hauptpunkt, dessen Vernachlässigung so entgegensetzte Urteile hervorbringt. Der Verstand und die Vernunft können alles vor ihren Richterstuhl fordern und machen leicht die Anmassung, das alles verständig, alles vernünftig sein solle, und somit entdecken sie freilich des Positiven genug, und das Schreien über Geistessklaverei, Gewissendruck, Aberglauben hat gar kein Ende ». Ibid., p. 142. — Un peu plus loin, Hegel affirme que ce qui, dans la religion, est uni, se trouve divisé par 
tendement qui est soumis à la critique, dans les textes francfortois, mais plus généralement le concept et la réflexion. Ce n'est plus la différence entre la Vernunft et le Verstand qu'utilise désormais Hegel pour comprendre la religion, mais l'opposition entre, d'une part, la réflexion ou le concept, source de division dans la réalité humaine, et, d'autre part, l'amour, la vie, l'esprit, principe d'unification des opposés ${ }^{54}$.

Cette absence, dans les textes de la période francfortoise, de la différence Vernunft-Verstand - différence qui, on le sait, réapparaîtra, et de façon définitive, dans les premières publications d'Iéna - offre une piste pour comprendre ce qui s'est passé dans l'esprit du jeune Hegel au cours de cette période, pendant laquelle prirent forme les concepts fondamentaux de sa philosophie. On ne saurait, bien sûr, apprécier le tournant qui s'opère alors sans tenir compte des influences exercées sur Hegel, celle de Hölderlin notamment ${ }^{55}$, celle aussi des lettres

la Vernunft «solche Zufälligkeiten, die dadurch, das etwas ewiges mit ihnen verknüpft ist, ihren Charakter der Zufälligkeit verlieren, haben deswegen notwendig zwei Seiten, und die Absonderung dieser zwei Seiten ist Trennung durch Vernunft». Ibid., p. 147.

54. Pour apercevoir cette opposition, omniprésente dans les textes de la période francfortoise, il suffit de rappeler comment, dans son essai sur L'esprit du christianisme et son destin, Hegel comprend le royaume de Dieu. «Im Reiche Gottes ist das Gemeinschaftliche, dass alle in Gott lebendig sind, nicht das Gemeinschafliche in einem Begriff, sondern Liebe, ein lebendiges Band, das die Glaubenden vereinigt, diese Empfindung der Einigkeit des Lebens, in der alle Entgegensetzungen, als solche, Feindschaften, und auch die Vereinigungen der bestehenden Entgegensetzungen ». Ibid., p. 321. «Die Entwicklung des Göttlichen in den Menschen, das Verhältnis, in das sie durch die Erfüllung mit dem heiligen Geiste mit Gott treten, seine Söhne zu werden, und in der Harmonie ihres ganzen Wesens und Charakters, ihren entwickelten Mannigfaltigkeit zu leben, einer Harmonie, in welcher nicht (nur) ihr vielseitiges Bewusstsein in einen Geist, die vielen Lebensgestalten in ein Leben einklingen, sondern durch welche auch die Scheidewände gegen andere gottähnliche Wesen aufgehoben werden, und derselbe lebendige Geist die verschiedenen Wesen beseelt, welche also nicht mehr nur gleich, sondern einig sind, nicht eine Versammlung ausmachen, sondern eine Gemeine, weil sie nich in einem Allgemeinen, einen Begriffe, etwa als Glaubende, sondern durch Leben, durch die Liebe vereinigt sind diese lebendige Harmonie von Menschen ihre Gemeinschaft in Gott, nennt Jesus das Königreich Gottes 》. Ibid.

55. Sur cette influence, voir D. HENRICH, op. cit., pp. 9-72, de même que la critique de H.S. HARRIS, op. cit., p. 294, note 2 . 
de Schiller sur l'Aestbetische Erziebung. Mais plutôt qu'une influence décisive venue de l'extérieur, c'est la maturation d'un idéal de raison qu'expriment sa rupture avec le kantisme et l'insistance sur la primauté de la vie et de l'amour sur la réflexion. L'abandon provisoire de la différence Verstand-Vernunft me parait traduire la prise de conscience par le jeune Hegel de l'insuffisance de l'idée de raison qui inspirait, à Tubingue et à Berne, sa critique de la religion. Définir la raison comme pouvoir de législation autonome et universelle, comme il le faisait dans les textes antérieurs, c'est oublier que la raison est principe d'unité dans la vie humaine, - ce dont pourtant il s'était rendu compte à Stuttgart et qu'il admirait le plus dans l'humanisme grec.

L'insuffisance de la raison, quand on la définit par sa seule fonction législatrice, consiste pour Hegel dans son impuissance à surmonter la division qu'elle introduit dans la réalité humaine. C'est dans L'esprit du cbristianisme que cette conviction se trouve formulée de façon la plus explicite, sous forme d'une critique du kantisme. Pour définir l'esprit nouveau introduit par Jésus, sa restauration de la subjectivité humaine contre l'objectivité du judaïsme, la façon kantienne d'interpréter le message évangélique dont Hegel s'était lui-même servi dans son Leben Jesu lui parait maintenant insuffisante. On pourrait s'attendre, remarque-t-il, à ce que, pour surmonter le légalisme de la religion juive, Jésus se soit appliqué à montrer que toute loi ici émane du sujet, du pouvoir de législation autonome et universelle qu'est la raison, et à enlever ainsi à la loi son objectivité et son hétéronomie apparentes. Mais une telle Aufbebung de la loi présuppose et consacre la division chez l'homme entre la raison et la sensibilité, entre l'universel du concept et la particularité des inclinations, entre l'objectivité et la subjectivité ${ }^{56}$. C'est par l'amour seulement que la loi peut être rendue superflue, car, étant l'actualisation du possible qu'elle énonce, il est le non-être de cette loi ${ }^{57}$. Avec l'amour,

56. NOHL, pp. $265-266$.

57. «Der völligen Knechtschaft unter dem Gesetzt einer fremden Herrn setzte Jesus nicht eine teilweise Knechtschaft unter einem eigenem Gesetzte, den Selbstzwang der kantischen Tugend entgegen, sondern Tugenden ohne Herrschaft und ohne Unterwerfung, Modifikationen der Liebe. \$ Ibid., p. 293. «Unmittelbar gegen Gesetzt gekehrt zeigt sich dieser uber Moralität er- 
on a affaire à quelque chose de simple, non plus à l'unité abstraite d'un concept; celui-ci, s'il est déterminé, exclut toute autre détermination, s'il est indéterminé, n'est rien de positif ${ }^{58}$. L'amour, ou la vie, dont il est une modification, est infini : non pas dans le sens où l'est une notion abstraite, car il s'agit d'un infini qui contient ses propres déterminations, d'un illimité qui se manifeste et se différencie par les limites qu'il se donne. Il est le premier nom, dans l'itinéraire hégélien, pour désigner l'universel concret, autour duquel s'organisera sa logique de l'absolu.

Si l'abandon par Hegel, dans les écrits de Francfort, de la différence entre Vernunft-Verstand, trahit une crise de pensée, il ne faut pas croire que celle-ci consista dans le rejet de son idéal de raison, dont il prit au contraire mieux conscience des implications. Selon cet idéal, tel qu'il le comprit avant sa lecture de Kant, la raison est principe de simplicité et d'harmonie. Or la comprendre comme pouvoir de législation subjective, par contraste avec l'entendement, faculté de règles objectives, ne lui suffit plus à exprimer cet idéal, car ainsi comprise, la raison, pas moins que l'entendement, introduit la division dans la vie entre l'universel et le particulier. Ce que Hegel a découvert à Francfort, c'est donc la nécessité de comprendre la raison comme esprit, comme une loi vivifiante - der Geist ist ein belebendes Gesetz in Vereinigung mit dem Mannigfaltigen - ${ }^{59}$ qui se différencie et

habene Geist Jesu in der Bergredigt, die ein an mehreren Beispielen von gesetzten durchgeführter Versuch its, den Gesetzten das Gesetztliche, die Form von Gesetzten zu benehmen, der nicht Achtung für dieselben predigt, sondern dasjenige aufzeigt was sie erfült, aber als Gesetzte, und also etwas Höheres is als der Gehorsam gegen dieselben und sie entbehrlich macht. Da die Pflichtgebote eine Trennung voraussetzen und die Herrschaft des Begriffs in einem Sollen sich ankündigt, so ist dagegen dasjenige, was über Trennung erhaben ist, ein Sein, eine Modifikation des Lebens, welche nur in Ansehung des Objekts betrachtet ausschliessend, also beschränkt ist, indem die Ausschliessung nur durch die Beschränkheit des Objekts gegeben ist und nur dasselbe betrifft. » Ibid., p. 266.

58. «Dies Einfache ist nicht ein negatives Einfaches, eine Einheit der Abstraktion, denn in der Einheit der Abstraktion ist entweder nut ein Bestimmtes gesetzt und von allen übrigen Bestimmtheiten abstrahiert, (oder) ibre reine Einheit ist nur die gesetzte Forderung der Abstraktion von allem Bestimmten; das negative Unbestimmte, reines Leben ist Sein». Ibid., p. 302-303.

59. NOHL, p. 347. 
se réalise à travers la multiplicité des gestes de l'homme. De l'idée de raison législatrice de l'expérience à l'idée de raison créatrice de ses propres déterminations, ou encore, de l'idée de raison qui n'introduit dans la vie qu'un Sollen auquel la nature doit être soumise à l'idée de raison qui est l'être absolu et qui «se modèle en nature par une force intérieure ${ }^{60}$, telle semble être la nouvelle étape effectuée à Francfort dans l'itinéraire hégélien.

S'il est vrai, comme l'a montré Pöggeler ${ }^{61}$, que Hegel est l'auteur du fragment Le plus ancien programme du système de l'idéalisme allemand, habituellement attribué à Schelling ou à Hölderlin, et que ce texte date du début de la période francfortoise, il faut avouer qu'il contient la formulation la plus explicite de cette nouvelle façon de comprendre la raison. "Je suis maintenant convaincu, écrit-il, que la fonction la plus haute de la Vernunft, celle qui comprend toutes les idées, est esthétique, et que la vérité et le bien ne trouvent leur parenté que dans le beau. Le philosophe doit, autant que le poète, possèder un pouvoir esthétique. Les hommes dépourvus de sens esthétique, ce sont nos philosophes livresques (unsere Buchstabenpbilosophen)» ${ }^{62}$. Ce n'est plus à Kant qu'il faut emprunter le langage pour parler de la raison, mais aux poètes, ou aux philosophes dotés comme eux d'un véritable sens esthétique.

60. Cette formule servira à Hegel dans le Differenzschrift à définir l'idéal de raison dont son époque a besoin : «...das Bedürfnis nach einer Philosophie hin, von welcher die Natur für die Misshandlungen, die sie in den Kantischen und Fichteschen Systeme leidet, wersöhnt und die Vernunft selbst in eine Uebereirstimmung mit der Natur gesetzt wird, - nicht in eine solche, worin sie auf sich Verzicht tut oder eine schale Nachhammerin derselben werden müsste, sondern eine Einstimmung dadurch, dass sie sich selbst zur Natur aus inner Kraft gestalet ». éd. H. BUCHNER.O. PÔGGELER, p. 8.

61. O. PÖGGELER, Hegel, der Verfasser des ältesten Systemprogramms des deutschen Idealismus, in Hegel-Studien 4, 1968, pp. 17.32. A la suite de G. SCHÜLER, Über Cbronologie von Hegels Jugendschriften, in HegelStudien, 2, 1963, pp. 111-161, H.S. HARRIS, op. cit.: p. 520, préfère situer ce texte au début de l'été 1796. On trouvera dans ce dernier ouvrage la présentation (pp. 249-257) et la traduction anglaise de ce texte (pp. 510$512)$.

62. «Ich bin nun überzeugt, dass der höchste Akt der Vernunft, der, in dem sie alle Ideen umfasst, ein aesthetischer Akt ist und dass Wahrheit und Güte nur in der Schönbeit verschwissern sind. Der Philosoph muss ebensoviel aesthetische Kraft besitzen als der Dichter. Die Menschen ohne aesthetischen Sinn sind unsere Buchstabenphilosophen ». J. HOFFMEISTER, Dokumente, p. 220 . 
Ce changement de perspective, dans lequel on aurait tort de voir un revirement brusque, impliquait pour le jeune Hegel de Francfort une façon nouvelle de comprendre la religion, différente de celle des Aufklarern. Il s'en explique de la façon la plus explicite dans la nouvelle introduction de 1800 à l'essai sur $L a$ positivité. Le procédé qui consiste à évaluer les comportements religieux particuliers à partir d'un concept abstrait de nature humaine, Hegel ne veut plus y avoir recours. L'emploi d'un tel concept oblige le critique à tenir pour contingent et superflu ce qui, dans la réalité, peut bien constituer l'essentiel et le plus beau. "Dans cet essai, écrit-il, je ne soumettrai pas la doctrine et la morale chrétiennes à la norme des concepts abstraits, ni ne jugerai si elles leur sont conformes ou non, si elles sont superflues, et par conséquent déraisonnables et inutiles. Le contingent, du fait que quelque chose d'éternel lui est attaché, perd son caractère de contingence et présente ainsi deux aspects qui, dans la religion, ne sont pas séparés: leur séparation n'est que l'oeuvte de la raison ${ }^{63}$. Sa nouvelle façon de comprendre la religion positive, le christianisme en particulier, va désormais se rattacher à ce que Hegel, dans le même passage, appelle «un beau présupposé », à savoir: «que tout ce qu'il y a de plus haut, de noble, de bon chez l'homme, est quelque chose de divin, issu de Dieu, son esprit, qui procède de lui ${ }^{64}$. Comprendre ainsi la religion, ce n'est plus la réduire à quelque chose de positif ; c'est la comprendre, comme il l'écrira à Iéna, de façon idéaliste. Tenir la religion pour une réalité positive, c'est ne pas voir qu'elle est une forme d'accomplissement de l'idéal d'humanité que chaque individu porte en lui, et qui n'est rien d'autre que le divin, l'absolu. Il n'y a pas que l'Aufklârer qui réduit la religion à sa positivité. C'est ce que fait également, et de façon criarde, quiconque sépare la nature humaine de Dieu, ou encore quiconque prétend qu'il n'y a qu'une seule médiation du divin, qui a eu lieu dans un individu,

63. NOHL, p. 143-144.

64. «Diese Ansicht des Verhältnisses der christlichen Religion zum Menschen ist nicht geradezu für sich selbst positiv zu nennen, sie bemerkt auf der gewiss schönen Voraussetzung, dass alles Höhere, alles Edle und Gute des Menschen etwas Göttliches ist, von Gott kommt, ein Geist ist, der von ihm ausgeht ». Ibid., p. 146. 
ou encore quiconque projette dans la foi en un être tout à fait étranger et tout-puissant la conscience que l'homme a du divin ${ }^{65}$.

Il est étonnant que des exégètes aient vu dans les écrits hégéliens de la période francfortoise le résultat d'une relecture de l'Évangile, plus chrétienne qu'à l'époque précédente où le jeune Hegel était encore retenu par le kantisme ${ }^{66}$. Car aussi bien à Francfort qu'à Tubingue et à Berne, il n'a cessé de chercher une forme de religion qui serait en harmonie avec son idéal de raison et qui en favoriserait le développement dans le peuple. Depuis l'essai sur la Volksreligion, il n'a jamais renoncé à exiger de la religion qu'elle soit issue de la raison, qu'elle touche le coeur et l'imagination du peuple et qu'elle inspire sa participation à la vie publique, et c'est toujours à la lumière de ces critères qu'il a examiné les religions juive et chrétienne et en est arrivé à refuser même la religion rationnelle proposée par les Aufklarem. C'est peut-être dans Le plus ancien programme du système de l'idéalisme allemand que se trouve le mieux définie la religion nouvelle que le jeune Hegel estime indispensable à tous les hommes, même les philosophes, et dont il souhaite la fondation. «Nous devons posséder une nouvelle mythologie, mais cette mythologie doit être placée au service des idées, elle doit devenir une mythologie de la Vernunft. Si les idées ne deviennent pas esthétiques, c'est-à-dire mythologiques, elles n'ont aucun intérêt pour le peu-

65. «Aber dann wird dies Ansicht zum grellen Positiven, wenn die menschliche Natur absolut geschieden wird von dem Göttlichen, wenn keine Vermittlung dersclben - ausser nur in einem Individuum zugelassen, sondern alles menschliche Bewusstsein des Gutten und des Göttlichen nur zur Dumpfheit und Vernichtung eines Glaubens an ein durchaus Fremdes Übermächtiges herabgewürdigt wird. $\gg I$ Ibid., p. 146.

66. "The revolution in Hegel's thinking came about, because, during his first two years in «unhappy Frankfurt» in order to cure himself of melancholia, he worked with all his energies at Greek literature and philosophy, as well as at history and politics, and then brought the result of these studies to bear anew on the reinterpretation of the life and message of Jesus... It was, however, his religious insight above all that scparared him from Kant. 》 T.M. KNOX, Hegel's Attitude to Kant's Ethics, in Kantstudien, 49 (1957), p. 71. - H. KÜNG, Menschwerdung Gottes. Eine Einfübrung in Hegels Tbeologisches Denken als Prolegomena zu eincr künftigen Cbristologie, Herder, 1970, p. 176, soutient qu'à l'époque de Francfort Hegel a fait faire un pas décisif à la philosophie moderne de la religion, par delà Kant ct l'Aufklärung, en se montrant disposé à reconnaître en Jésus le Fils de Dieu. 
ple; si au contraire la mythologie ne devient pas rationnelle, le philosophe doit en avoir honte. De sorte que tous, éclairés ou non, doivent se donner la main pour rendre la mythologie philosophique, le peuple raisonnable, et la philosophie mythologique, les philosophes sensibles ${ }^{67}$. Cette religion, on le sait, ne pourra être instaurée, selon l'esprit de l'auteur de ce fragment, qui semble bien être de Hegel, que par la raison, dans sa fonction la plus haute, la fonction esthétique.

Plutôt qu'une conversion religieuse, les textes de Francfort révèlent donc un changement dans la façon hégélienne de comprendre la raison. Le tournant pris par Hegel, durant les quatre dernières années de préceptorat, a consisté avant tout dans l'insatisfaction devant la théorie kantienne de la raison et dans la recherche d'une compréhension plus juste de sa fonction propre. Il s'est rendu compte que le kantisme s'en est tenu, sur l'objet de la raison, à une conception vague et négative. L'infini pensé par la raison kantienne n'est qu'un simple au-delà du fini; dans l'idée elle ne pose rien d'autre qu'un devoir-être, un Sollen. C'est cette insatisfaction que traduit le refus de Hegel, à cette période, d'utiliser l'opposition Verstand-Vernunft, qu'il savait étroitement liée au kantisme et c'est la recherche d'une compréhension plus juste de la raison qu'exprime l'insistance des textes de cette période sur la primauté de la vie, de l'amour et de l'esprit. De ce refus et de cette recherche résulte un nouveau concept de raison, qui ne la définit plus par l'autonomie et l'universalité de sa législation, mais la comprend comme l'universel concret, l'identité concrète du sujet et de l'objet, du concept et de l'être.

De l'analyse des Jugendschriften, on peut tirer deux conclusions, modestes mais utiles à l'intelligence de la théologie hégé-

67. «Zuerst werde ich hier von einer Idee sprechen die, soviel ich weiss, noch in keines Menschen Sinn gekommen ist - wir müssen eine neue Mythologie haben, diese Mythologie aber muss im Dienste der Ideen stehen, sie muss eine Mytbologie der Vernunft werden. Ehe wir die Ideen äesthetisch, d.h. mythologisch machen, haben sie für das Volk kein Interesse; und ungekehrt, ehe die Mythologie vernünftig ist, muss sich der Philosoph ihrer schämen. So müssen endlich Aufgeklärte und Unaufgeklärte sich die Hand reichen, die Mythologie muss philosophisch werden und das Volk vernünftig, und die Philosophie muss mythologisch werden, um die Philosophen sinnlich zu machen ». Dokumente, p. 221. 
lienne. S'il est vrai, en premier lieu, que les travaux de jeunesse ne servent guère à comprendre l'attitude de Hegel à l'égard du christianisme, puisqu'ils appartiennent à une période où il ne voit pas encore dans le christianisme la religion absolue, ils sont d'un très grand secours pour la reconstitution d'une position philosophique indispensable à l'élaboration de la Religionspbilosophie: la façon de comprendre la raison dans son contraste avec l'entendement. En deuxième lieu, la lecture des Jugendschriften permet, sinon de dévoiler l'intentionnalité de la théologie hégélienne, du moins de comprendre le rapport entre la logique de cette théologie et la révélation chrétienne. Prétendre, comme on l'a fait, que la logique hégélienne de l'Absolu a sa source dans la révélation, du fait qu'elle présente ce que Dieu doit être pour pouvoir se révéler et qu'elle reçoit ce contenu de la révélation elle-même ${ }^{68}$, c'est à mon avis méconnaître que dans sa structure fondamentale cette logique de l'Absolu s'imposa à Hegel à une époque où le christianisme ne représentait pas pour lui la religion absolue. On s'aperçoit plutôt, si l'on suit la méditation de Hegel sur la différence entre la Vernunft et le Verstand, que cette logique a été le résultat d'un débat avec le kantisme, ou mieux le fruit de l'attachement du jeune Hegel à un idéal de raison dont il a pris conscience progressivement. Et l'on reconnait en outre ce que cette logique lui offrait: la possibilité de définir ce que Dieu doit être pour que la raison humaine puisse se maintenir dans son immanence et sa liberté.

On voit le problème posé par la philosophie hégélienne de la religion : celui de la possibilité d'une théologie de la révélation pour une raison qui se comprend de telle façon que recevoir d'autrui représente pour elle une infraction à sa spontanéité. Toute philosophie de la religion vise à articuler la rationalité vécue par la foi religieuse; son oeuvre consiste à poser et à maintenir le cogito de cette foi. La philosophie hégélienne de la religion, en dépassant la dissociation antinomique de l'intelligence opérée par la philosophie critique, et en restaurant une vraie vie spécula-

68. «La logique hégélienne de l'Absolu présente ce que Dieu doit être pour qu'il puisse se révéler, mais c'est la révélation elle-même qui offre ce contenu ». C. BRUAIRE, op. cit., p. 21. 
tive de la pensée, s'est crue en mesure d'assimiler intégralement la foi religieuse, d'en reprendre les enseignements à l'intérieur du savoir spéculatif auquel elle s'élève. On peut difficilement, lorsqu'on veut méditer les leçons d'une philosophie de la religion, se référer à un modèle plus parfait. Car dans la raison telle qu'il la comprenait, Hegel voyait la synthèse de la subjectivité moderne et de l'objectivité de la tradition ontologique, la conciliation de la certitude et de la vérité. En elle il croyait à la possibilité de récupérer les acquis intellectuels de la foi chrétienne et de ses élaborations théologiques. L'idéal hégélien de la raison réussit-il à fournir le cogito de la foi chrétienne? Quelle compréhension de l'intelligence peut constituer l'inaliénable d'une philosophie soucieuse de se procurer une assise rationnelle capable de respecter dans leur intégrité, tout en intégrant leur vérité propre, les discours respectifs de la science et de la foi ? Si l'étude de la théologie hégélienne n'a pas grand chose à apprendre au croyant sur le contenu de sa foi, elle peut lui enseigner, et de façon magistrale, une façon possible de déployer ce contenu en un discours rationnel articulé.

Université d'Ottawa 